\title{
Effects of combination pharmacotherapy and social skills training for schizophrenia: A randomized controlled trial*
}

\author{
Eiko Shimada $^{1}$, Nobuo Anzai ${ }^{2}$, Emi Ikebuchi ${ }^{3}$, Shin-Ichi Niwa ${ }^{4}$, Masahisa Nishizono ${ }^{5}$ \\ ${ }^{1}$ Department of Psychology, Bunkyo Gakuin University, Fujimino, Japan \\ ${ }^{2}$ Department of Clinical Psychology, Faculty of Health and Medical Science, Teikyo Heisei University, Tokyo, Japan \\ ${ }^{3}$ Department of Psychiatry, Teikyo University, School of Medicine, Tokyo, Japan \\ ${ }^{4}$ Department of Psychiatry, Aizu Medical Center, Fukushima Medical University, Fukushima, Japan \\ ${ }^{5}$ Institute for Psycho-Social Psychiatry, Fukuoka, Japan \\ Email: eikoshimada@mtf.biglobe.ne.jp,nanzai@nifty.com, PXM06766@nifty.ne.jp, si-niwa@,fmu.ac.jp, zva11061@nifty.ne.jp
}

Received 26 April 2013; revised 25 May 2013; accepted 3 June 2013

Copyright (C) 2013 Eiko Shimada et al. This is an open access article distributed under the Creative Commons Attribution License, which permits unrestricted use, distribution, and reproduction in any medium, provided the original work is properly cited.

\begin{abstract}
Pharmaco-psychosocial treatment (PPST) refers to a treatment paradigm combining pharmacotherapy with psychosocial therapy. We conducted an 18-month randomized controlled tracking study to evaluate the efficacy of social skills training (SST) in 11 individuals with schizophrenia on risperidone monotherapy. They were randomized to either SST or non-SST group, and Global Assessment Functioning (GAF) and Brief Psychiatric Rating Scale (BPRS) scores in the 2 groups showed gradual improvement. In the SST group, Digit Span Distraction Test (DSDT) at 12 and 18 months showed significant improvement. The Subjective Deficit Syndrome Scale (SDSS) scores in the SST group showed significant improvement after 6 months and showed a significant difference in comparison between the groups. After the subjective feeling of health was recovered, the items of motivation were recovered as a direct effect of SST. Some items of the Life Assessment Scale for the Mentally Ill (LASMI) score showed significant improvement (or significant trend) in only the SST group after 6 months. Items of daily life, work skills, and self-recognition were continuously improved. We report 2 cases in the SST group that had DSDT improvement in parallel with change of action and communication as a change of social function. This study is only exploratory due to the small number of cases. However, we have demonstrated potential improvement in cognitive function due to learning experiences that required working memory and attention, thus providing improvement in social life.
\end{abstract}

*Conflict of interest: The authors have no potential conflicts of interest relating to the subject of this report.
Keywords: Schizophrenia; Social Skills Training; Randomized Controlled Trail; Cognitive Function; Risperidone

\section{INTRODUCTION}

The pharmaco-psychosocial treatment (PPST) research group, which was proposed by Nishizono in 2000 [1], aimed to promote treatments combining pharmacotherapy and psychosocial treatment for schizophrenia. The PPST research group promotes educational activities throughout Japan. However, there is not yet sufficient evidence for the efficacy of this treatment.

Social skills training (SST) is a therapeutic component that is provided alone or with many other therapies, which when carefully combined, can increase prospects for recovery of individual with mental disorder [2]. It is difficult to numerically express the small changes in efficacy resulting from SST. In Patient Outcome Research Team (PORT) guidelines and the American Treatment of Schizophrenia guidelines, SST and cognitive therapy are suggested as maintenance therapies, and their effectiveness has been confirmed [3].

In this study, we report the results of an 18-month study of 11 cases who were separated into a pharmacotherapy group and a pharmacotherapy with SST group and followed up to examine the clinical effectiveness of PPST. We report results comparing both groups.

\section{SUBJECTS AND METHODS}

The subjects were outpatients with schizophrenia diagnosed according to the DSM-IV-TR criteria. All subjects under 40 years of age, had received risperidone (RIS) continuously for at least 8 weeks, and had never 
received SST until the start of this study. All of them agreed to participate in the study and provided written informed consent. This study was conducted in accordance with the ethical principles listed in the 1964 Declaration of Helsinki and with the approval of the eth- ics committees of our hospitals.

These subjects were randomly assigned to either the SST group or the non-SST group. Eleven cases (7 men and 4 women) were assigned to the SST group ( 6 cases; age, $31.7 \pm 7.9$ years, mean \pm standard deviation [SD]) and 5 to the control group (age, $30.6 \pm 8.2$ years). All patients received RIS monotherapy ( $\leq 12 \mathrm{mg} /$ day) as antipsychotic maintenance therapy, and cases in the SST group received SST concomitantly for at least 6 months. Cases in the SST group participated in a course of medication self-management (about $1 \mathrm{~h} /$ week), which was a module of the SST program that was completed over approximately 9 months. Doctors, nurses, and a pharmacist served as SST trainers for this module. We practiced how to communicate in accordance with the basic training model, and we practiced 4 skills: to obtain information about the benefits of antipsychotic medication, to know the correct method of self-administration and evaluate medicine, to identify the side effects of medication, and to negotiate medication issues with health-care providers. Continuing the training, they played the roles of doctor, nurse, and caseworker and practiced asking about benefits and side effects of the medicine in a consultation room or by telephone. They also practiced how to consult and confirmed their knowledge about the medication. They practiced making appointments and consulting with providers. They practiced how to handle the situation if they ran out of medication or had insufficient medication, and how to handle invitations of drinking, by using problem-solving methods. All cases were followed up for 18 months and received clinical evaluation at study entry and at $3,6,12$, and 18 months thereafter.

\section{OBJECTIVE ASSESSMENTS}

\subsection{Global Assessment Functioning}

The Global Assessment Functioning (GAF) considers social and occupational functioning on a hypothetical continuum of mental health disorders, which includes impairment in functioning due to physical limitations, as well as mental impairment. Producing the GAF rating involves choosing single values that best reflect the individual's overall level of functioning. The description of each 10-point range in the GAF scale has 2 components: the first part covers symptom severity and the second part covers functioning. The GAF rating is within a particular decile if either the symptom severity or the level of functioning falls within the range.

\subsection{Brief Psychiatric Rating Scale}

The Brief Psychiatric Rating Scale (BPRS) was developed to provide rapid assessment of severity of schizophrenic states during treatment. It consists of 18 items of mostly global clinically familiar symptoms and behavior constructs that span much of the range of manifest psychopharmacology, each rated on an 8-point scale. Ratings are made during a brief (15 - $20 \mathrm{~min})$, unstructured interview with the individual with mental illness. Each item is defined by 1 or 2 sentences of clinical description $[4,5]$.

\subsection{Drug-Induced Extrapyramidal Symptoms Scale}

The Drug-Induced Extrapyramidal Symptoms Scale (DIEPSS) was used to assess treatment-emergent extrapyramidal symptoms. It consists of 8 individual parameters and 1 global assessment for the purpose of estimating extrapyramidal symptoms as an adverse event. The severity of each item is graded from 0 (normal) to 4 (severe), and each category has clearly defined anchor points [6-8].

\subsection{Life Assessment Scale for the Mentally Ill}

The Life Assessment Scale for the Mentally Ill (LASMI) was used to evaluate how schizophrenia affects every day social life in diverse life situations. It consists of daily life (12 items), interpersonal relations (13 items), work skills (10 items), endurance and stability ( 2 items), and self-recognition (item 3) from a total of 40 items in 5 subscales. Score is 0 points (no problem) to 4 points (much of a problem). A higher score means that the symptoms are severe $[9,10]$.

\subsection{Digit Span Distractibility Test}

The Digit Span Distractibility Test (DSDT) was used to evaluate cognitive function. It is a digit span task under an interference condition, and is one of the methods used to examine neurocognitive function. Healthcare professionals conduct the test with an attached CD-ROM, and individuals with mental illness write the answers on an answer sheet. The evaluator (healthcare professional) counts the number of correct answers (number of digits correctly in order) and the number of erroneous answers, and calculates the percentages of correct answers [11, $12]$.

\section{SUBJECTIVE ASSESSMENTS}

\subsection{Medical Outcome Study Short Form 36 Health Survey (SF-36)}

The SF-36 is a generic measure of functional health and 
well-being with psychometrically based physical and mental health summary measures, comprising 8 domains that assess quality of life parameters. Scores range from 0 to 100 , with higher scores indicating a better quality of life. From these 8 domains, a physical component summary score and a mental component summary score were calculated [13-15].

\subsection{Subjective Deficit Syndrome Scale (SDSS)}

The Subjective Deficit Syndrome Scale (SDSS) consists of 19 items that constitute subjective complaints. It is intended to measure only self-reported complaints of the individual with schizophrenia, and not the interviewer's judgment of the individual's distress [16-18].

All assessment results were analyzed statistically.

\section{RESULTS}

None of the cases in the SST group dropped out during the 18 months because of aggravated symptoms or adverse reactions. At the entry of this study, the average dose of RIS (mean \pm SD) of the SST group was $6.8 \pm 3.9$ $\mathrm{mg}$ /day, while that of the non-SST group was $2.2 \pm 1.3$ $\mathrm{mg} /$ day. There was no significant difference in the RIS dosage between the 2 groups $(p<0.05)$. At the 18 -month follow-up, the average dose of RIS of the SST group was $7.0 \pm 2.4 \mathrm{mg} /$ day, while that of the non-SST control group was $4.0 \pm 1.9 \mathrm{mg} /$ day. There was no significant difference in the RIS dosage between the 2 groups $(p<$ $0.05)$. There was an increasing tendency of RIS dosage at the beginning of the intervention and at the 18th month in the SST group.

Data of both groups showed a tendency toward improvement in the rating scales during the follow-up. The summary of the results of this study is as follows:

1) The GAF and BPRS scores trended toward modest improvements in both groups. Only the SST group showed significant improvement in the GAF score compared with that at the start (Figure 1). The SST group showed significant improvement in the total BPRS score, while the non-SST group showed a nonsignificant improvement (Figure 2).

2) The SDSS also showed significant improvement after 6 months, and showed a significant difference when both groups were compared (Figure 3). In the SDSS items, "Insomnia", "Impoverished motivation", "Vegetative disorder", and "Sensitivity to weather" showed improvement after 6 months, and "Loss of emotions/indifference" showed improvement after 12 months. This shows that a subjective feeling of health was restored first, followed by recovered motivation and other qualities. It is possible that this was a direct effect of SST.

3 ) In the longitudinal course of 18 months, the DSDT score showed a significant improvement in the SST

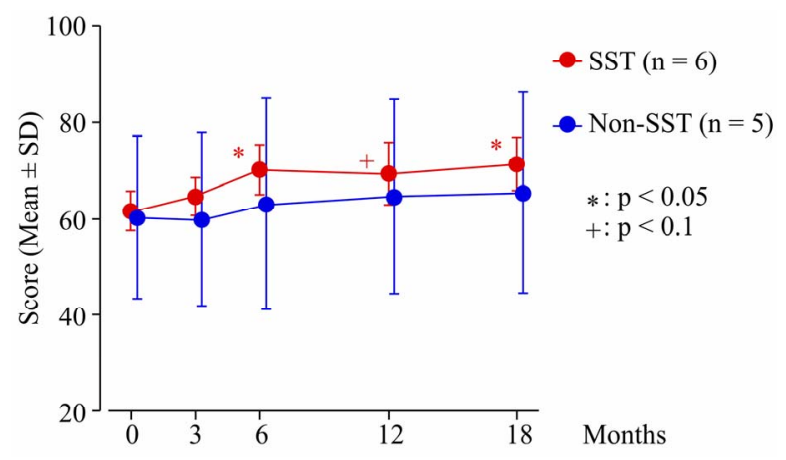

Figure 1. Changes in GAF (Global Assessment Functioning) Scale scores over 18 months. The red circles represent the average scores of the SST (social skills training) group (received SST concomitantly). The blue circles represent the average scores of the non-SST group (did not receive SST). The higher the GAF score, the severe are the symptoms. Within each group, changes were tested with Wilcoxon signed-rank test. Group comparisons of change score were tested with U-test. The SST group alone showed a significant improvement in the GAF score compared with that at the start: at the 6th $(p<0.05), 12$ th $(p<0.1)$, and 18th $(p<0.05)$ months.

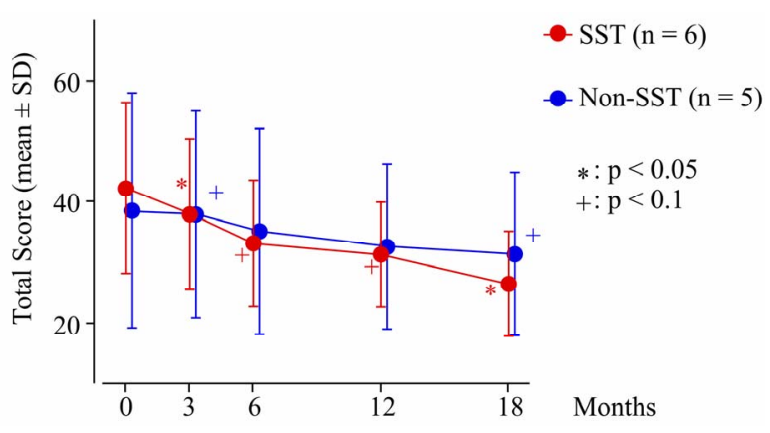

Figure 2. Changes in BPRS (Brief Psychiatry Rating Scale) scores over 18 months. The red circles represent the average scores of the SST (social skills training) group (received SST concomitantly). The blue circles represent the average scores of the non-SST group (did not receive SST). The higher the BPRS score, the severe are the symptoms .Within each group, changes were tested with Wilcoxon signed-rank test. Group comparisons of change score were tested with U-test. The SST group showed a significant improvement in the total BPRS score at the 3rd $(p<0.05)$, 6th, 12th $(p<0.1)$, and 18 th $(p<0.05)$ months.

group at the 12th and 18th months (Figure 4), but there was no significant difference in either group.

4) Some items in LASMI showed significant improvement or a significant trend only in the SST group after 6 months of treatment (Figure 5). "daily life (D)", "work skills (W)", and "self-recognition (R)" specifically showed continued improvement.

5) There was no significant difference in the RIS dosage from the beginning of the intervention and at the 18th month time point in the SST group. However, DIEPSS after 12 months and 18 months tended to improve. 


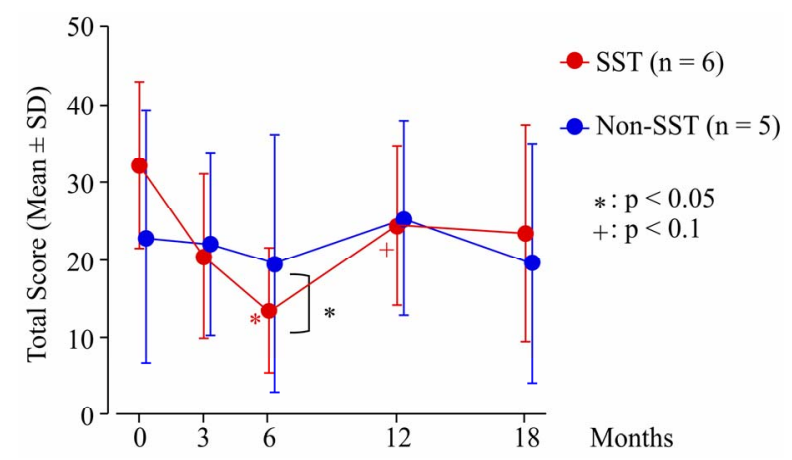

Figure 3. Changes in SDSS (Subjective Deficit Syndrome Scale) scores over 18 months. The red circles represent the average scores of the SST (social skills training) group (received SST concomitantly). The blue circles represent the average scores of the non-SST group (did not receive SST). The higher the SDSS score, the severe are the symptoms .Within each group, changes were tested with Wilcoxon signed-rank test. Group comparisons of change score were tested with U-test. The SST group also showed a significant improvement at the 6th $(p<0.05)$ and 12th $(p<0.1)$ months. A significant difference was found when both groups were compared at the 6th month $(p<0.05)$.

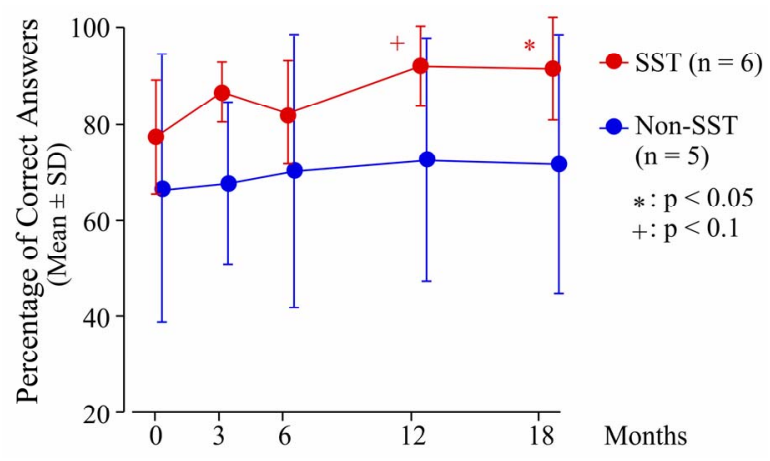

Figure 4. Change in the percentage of correct answers on the DSDT (Digit Span Distractibility Test) over 18 months. The red circles represent the average scores of the SST (social skills training) group (received SST concomitantly). The blue circles represent the average scores of the non-SST group (did not receive SST). Within each group, changes were tested with Wilcoxon signed-rank test. Group comparisons of change score were tested with U-test. Percentage of correct answers $=($ Number of correct answers - Number of incorrect answers $) \div($ Number of target digit numbers $) \times 100(\%)$. The SST group showed a significant improvement in the DSDT score at the 12th $(p<0.1)$ and 18th $(p<0.05)$ months.

\section{DISCUSSION}

\subsection{Characteristics of This Study}

Our study has some clear trends. It is particularly worth mentioning that this was a randomized controlled study and there was a follow-up investigation for 18 months from the start of the treatment in order to clarify the effect of SST on social function. Kurtz et al. performed a metanalysis of clinical studies on SST randomized con- trolled trials [19]. They investigated 1521 cases in 22 studies and showed that the effect size on acquisition of practiced skills was large $(d=1.20)$; the effect size on improvement of general social skills, the skills of daily life, and the skills of community life were modest $(d=$ 0.52 ); and the effect size on other symptoms and recurrence prevention were small $(d=0.40)$. These results show what cases can obtain directly by SST, and they show that patients need a comprehensive treatment program to get a broader range of outcomes. These effect sizes in many studies were measured soon after SST intervention $[20,21]$. It is clinically very important to investigate what social functions are acquired in the long term by acquiring skills for communication and daily life. It is important to select cases who have motivation to participate in psychosocial treatment. Without the consent of the cases to participate fully, it is hard to maintain participation and to assess efficacy, which makes it difficult to conduct randomized controlled trials. Our study on the effect of SST was the first random allocation in Japan.

\subsection{Changes in Individual Participants before and after SST Intervention}

The statistical power was insufficient because the number of cases was small. We verified the changes in the groups by using individual cases and compared them with cases in the control group.

\subsection{Case A: A 39-Year-Old Man (Figure 6)}

The GAF and BPRS scores improved, and the DSDT and LASMI scores mildly improved. The GAF score was 55 at study entry, 58 at the 3rd month, 80 at the 6 th month, and continued improving until the end of the study. The DSDT score was $61 \%$ at study entry and $97 \%$ at the 18 th month. In the LASMI, the scores of daily life (D) and self-recognition (R) improved. This corresponds to the fact that he was dependent on his mother and he did not clean his room well at the start of SST, and that he had begun paying attention to his life rhythm and appearance by himself at the 18-month follow-up exam. As he improved his skills by the medication self-management module, he could tell a doctor that he was able to sleep well. As a result, his dose of hypnotics was reduced. Although he was reluctant to take medicine until then, he realized that he slept well and began to participate in treatment positively.

The medication self-management module also helped him, in that it allowed him to bring a medical certificate to the government office of disability pension. The practice of taking notes played an important role in organizing his daily life, and allowed him to acquire the skills to speak to his doctor by checking his notes during consul- 


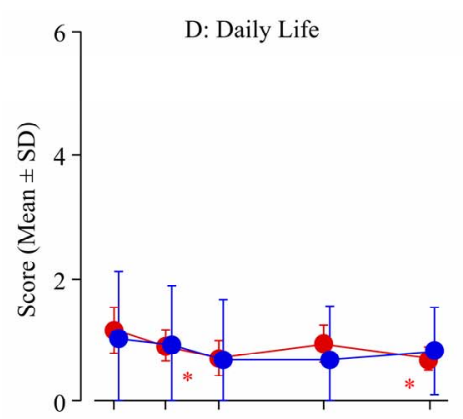

Months

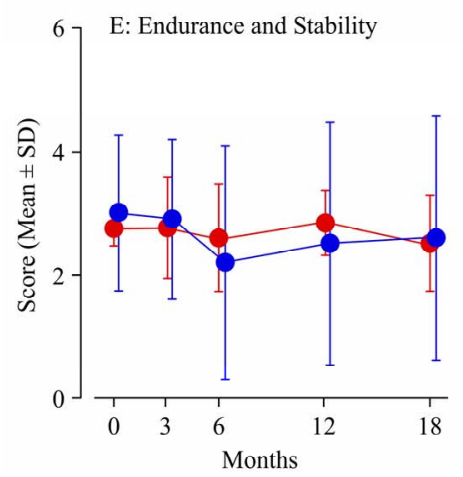

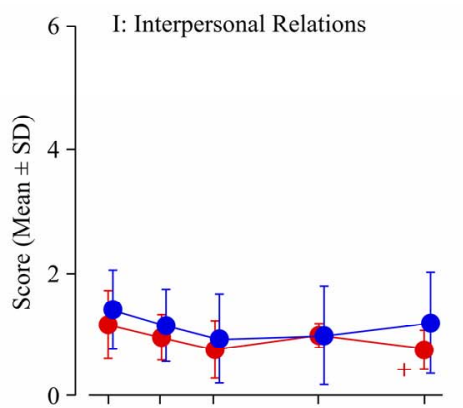

Months

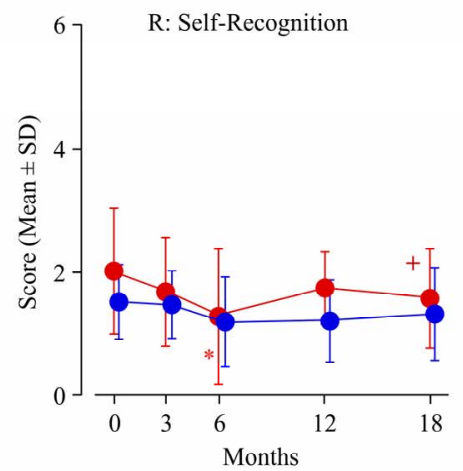

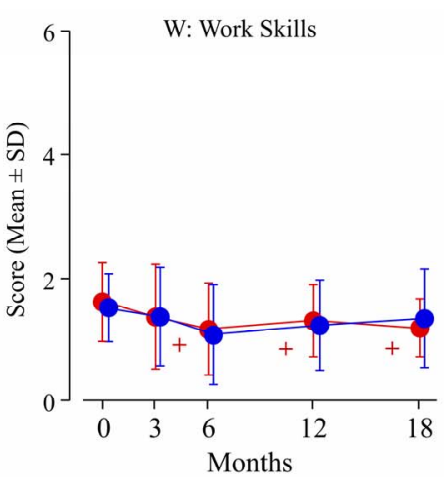

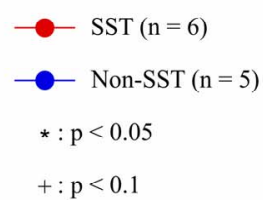

Figure 5. Change in the LASMI (Life Assessment Scale for the Mentally III) scores over 18 months. The red circles represent the average scores of the SST (social skills training) group (received SST concomitantly) on the subscales of the LASMI. The blue circles represent the average scores of the non-SST group (did not receive SST) on the subscales of the LASMI. The higher the LASMI score, the severe are the symptoms. Within each group, changes were tested with Wilcoxon signed-rank test. Group comparisons of change score were tested with U-test. Some items in the LASMI showed a significant improvement or a significant trend only in the SST group: "daily life (D)", at the 6th and 18th months ( $p<0.05)$; "interpersonal relations (I)", at the 18th month $(p<0.1)$, "work skills (W)", at the 6th, 12th, and 18th month $(p<0.1)$; and "self-recognition (R)", at the 6th $(p<0.05)$ and 18 th $(p<0.1)$ months.

tation. He could fill in a medication checklist and report regularly. As he continued to practice his medication list (to write the date on a pill bag and to arrange pills in each week), he became more skilled at this task. He wrote a daily medication management table to report during his examinations. In the medication self-management knowledge question (yes-no question), he scored 17 of 19 (89\%) before training and had an improved score of 19 points $(100 \%)$ after training. Thus, although the evaluation scales did not improve greatly, his skills for social life improved in many aspects. His job of 7 years was to compounding materials for house walls, which involves weighing, mixing, and packing materials (X kg of material A and $\mathrm{Y} \mathrm{kg}$ of material B and etc.). The fact that the SDSS score became worse at the 12th and 18th months may correspond to the fact that he had complained to the factory manager where he worked that his workload was increased, and he had quarreled with his mother after 8 months. He seemed to feel stressed because he had not been accustomed to dealing with his work efficiently and systematically.

After his amount of work was decreased, he relaxed and his symptoms improved. He became better able to be aware of his fatigue, but he was poor at making arrangements. Subsequently he gained strength and began to consult about his troubles. He began to rest well on weekends. Initially, he had difficulty in systematically proceeding to plan and carry out a number of challenges, but we found that there was significant improvement in his efficiency, so he had difficulty in performing tasks and working according to plan at first, but with treatment, he began to work more effectively. This shows that his attention also improved. When the factory manager spoke to workers, he initially felt accused by the manager. However, he learned to understand that the manager spoke not just to him, but to others workers also.

We compared this case with a 34-year-old man in the conventional treatment group (non-SST group), who was near the same age. His LASMI score and SDSS score did not improve. The reasons might be as follows: He was married and he was a former dental technician whose social skills had been high. His schizophrenia symptoms had started 19 months before baseline assessment. Because he had paranoid ideation and exhibited behaviors 

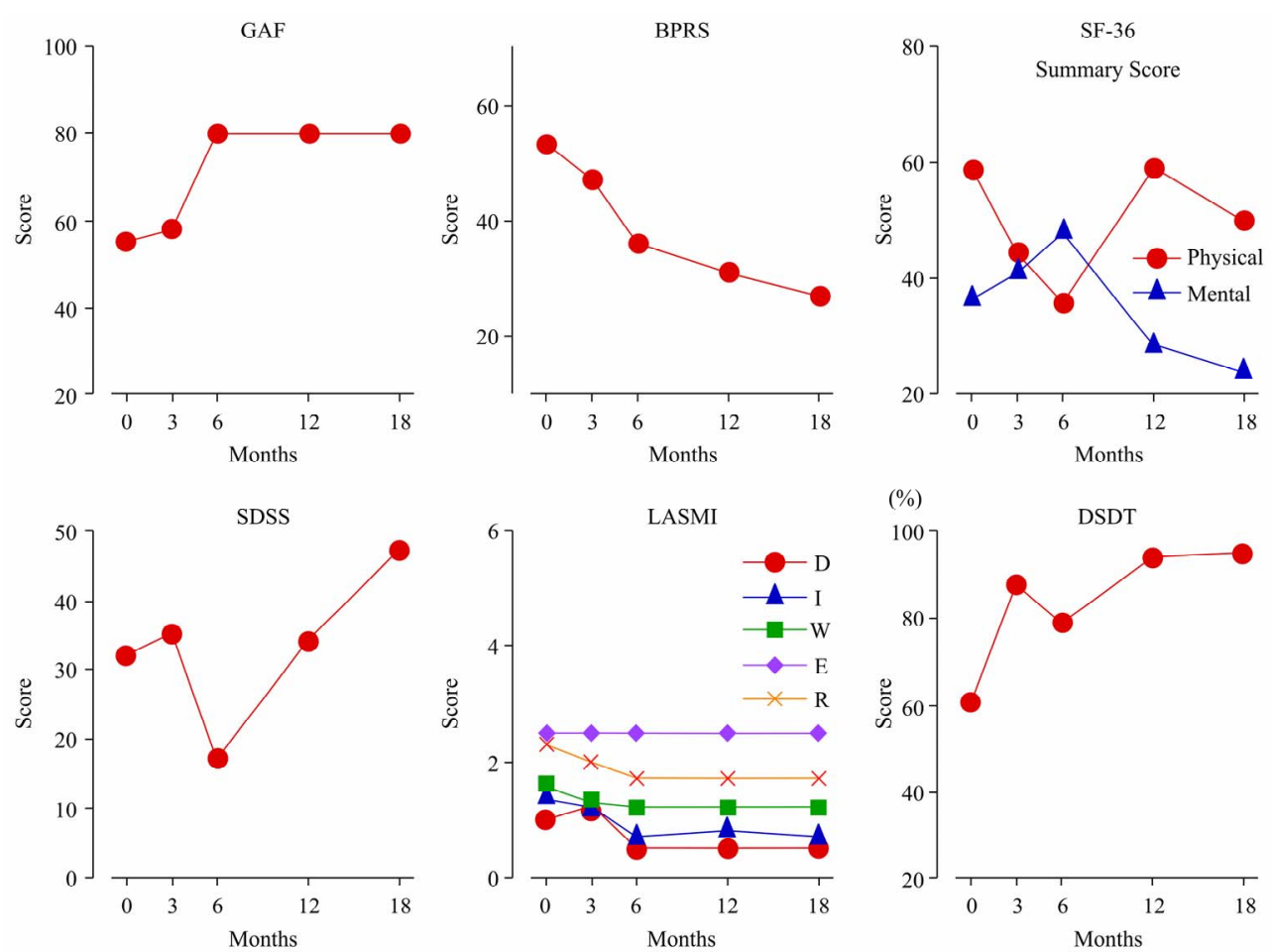

Figure 6. Case A (39-year-old man, received SST concomitantly) in the longitudinal course over 18 months. The red circles represent the scores on GAF, BPRS, SDSS, and DSDT. In the SF-36 (MOS Short-Form 36-Item Health Survey), the red circles represent the summary scores on the physical component and the blue triangles, the summary scores on the mental component. In the LASMI, the red circles represent "daily life (D)"; blue triangles, "interpersonal relations (I)"; green squares, "work skills (W)"; purple diamonds, "endurance and stability (E)"; and yellow crosses, "self-recognition (R)". GAF and BPRS scores improved, and the DSDT and LASMI scores improved slightly. The GAF score was 55 at the study entry, 58 at the 3rd month, 80 at the 6th month, and continued to improve until the end of the study. The DSDT score was $61 \%$ at the study entry and $97 \%$ at the 18 th month. In the LASMI, the scores of daily life (D) and self-recognition (R) improved.

threatening to self and others (i.e., reckless speeding on expressways), he was committed to inpatient treatment, and subsequently he improved. Superficially, his functions did not decrease significantly, and a relatively small dosage of RIS was effective. Initially, for restlessness and anxiety, he took lorazepam, but he gradually decreased taking this medication. At the end of the study, he quit his job as a dental technician and began to go to nursing school. His DSDT score improved temporarily, but became worse as time passed. At the beginning, he sometimes forgot to take medicine in the evenings. This meant that his adherence of medication and insight was poor. With respect to LASMI, he had a social goal such as going to a nursing school, but his life rhythm scores, such as daily life (D) did not change, and handling of vagueness and resistance to stress did not improve over the long term during this study. The SDSS scores also were unchanged.

\subsection{Case D: A 38-Year-Old Man (Figure 7)}

In this case, the subject's GAF score and BPRS score improved and the DSDT score and LASMI score improved slightly as time passed. The SDSS score improved significantly at the $3 \mathrm{rd}$ month, and continued to improve. With reduced RIS dosage $(11 \mathrm{mg} /$ day at the study entry, $6 \mathrm{mg} /$ day at the 18 th month), the DIEPSS score improved significantly.

In SST role-play, facial expression and nonverbal communication improved. In fact, the case, came to the clinic with a smile every time, although he still reported hearing voices. He could fill in the medication check list every day, which he practiced in the medication self-management module, and could report regularly. He did not attend the daycare treatment regularly. He was obese, and his blood test results showed fatty liver. After attending SST, he was able to go to the daycare center several days a week at the 6th month, but he complained of insomnia due to interpersonal tension at the daycare treatment. Until the 18th month, he found that his delusion and auditory hallucinations decreased. He began a diet (not exercise, but mainly meal and beverage restrictions). As a result, he lost about $10 \mathrm{~kg}$ of body weight and his blood test results improved. He was able to ask 

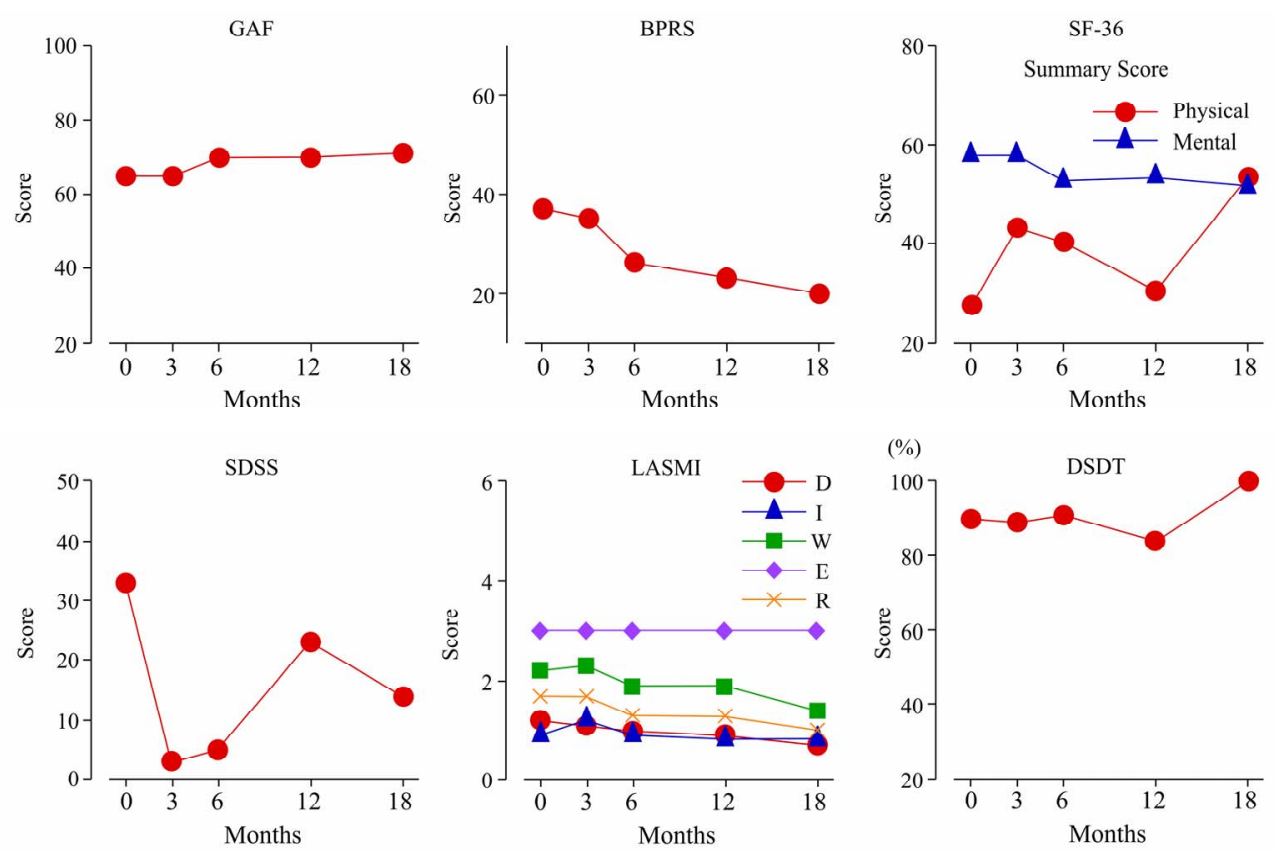

Figure 7. Case D (38-year-old man) in the longitudinal course over 18 months. The red circles represent the scores on GAF, BPRS, SDSS, and DSDT. In the SF-36 (MOS Short-Form 36-Item Health Survey), the red circles represent the scores on the physical component and the blue triangles represent the total scores on the mental component. In the LASMI, the red circles represent "daily life (D)"; blue triangles, "interpersonal relations (I)"; green squares, "work skills (W)"; purple diamonds, "endurance and stability (E)"; and yellow crosses, "self-recognition (R)". Over time, the GAF and BPRS scores improved and the DSDT and LASMI scores improved slightly. The SDSS score improved significantly at thee $3 \mathrm{rd}$ month and continued to improve thereafter.

the doctor to reduce the amount of his hepatoprotective medicine because he lost weight and his hepatic function improved, and his attitude towards consultation changed. He became active and he attended daycare 4 days a week and did not complain of fatigue. His body shape significantly changed. These improvements in his condition seemed to affect his SDSS score.

At the start of the study, the case had severe syndromes regarding nervousness, lack of stamina, tolerance for conflicts, external events, impoverished motivation, and willpower/initiative, but all items became mild at the 3 rd and 6th months. At the 12th month, he reported that he often felt physical/mental lassitude, adynamia, and lack of stamina. He reported insomnia and he was worried about a friend at the daycare services at that time. However, he solved his problems and these items improved. Because there were changes in relationships with friends at the daycare and he had interaction with new SST members, he paid attention to his appearance and his hair style. These facts affected improvements in his establishment of life rhythm, balanced diet, money management, and dosage management in daily life (D); spontaneous conversation and rejection in interpersonal relations (I); challenge to problem, prospects for achievement, understanding of procedures, alteration of procedure, stability, and resistance to stress in work skills (W), and some items in self-recognition (R).

He had been dependent on his aged father to pick him up when he had consultations with his doctor and when he attended the daycare services. He began to get himself ready and go alone by bus. Moreover, he liked the status quo and disliked changes. He had no future goals at first, but then he began to look forward to going to the workplace.

Here, we compared the individual in case D with a woman in the conventional treatment group (non-SST group) who was 33 years old. Her DSDT score did not improve, but her SDSS score and LASMI score improved slightly as time passed. She had no children and had been a housewife for a long time. Because she suffered from auditory hallucinations and conflict with work peers, she quit her part-time job at a supermarket. She had few friends and secluded herself in her house. Her auditory hallucinations improved with increased dosage of RIS. There were a few social changes, but she was able to have a stable family life with her husband. She was essentially sensitive to her change in physical condition. It is possible that because she quit her job and she did not need to make cumbersome associations with her work peers, her physical condition and fatigue improved, so she became well and her SDSS score improved. 


\subsection{Improvement Tendency of the DSDT}

In the DSDT, individuals with mental illness need to ignore noticeable stimuli, focus on target stimuli, and memorize and dictate displayed numbers. This test is used for testing selective attention and verbal working memory [22].

The DSDT score showed significant improvement in the SST group at the 12th and 18th months (Figure 4), but there was no significant difference in either group. It is possible that the statistical power was insufficient because the number of cases was small, and cases in both groups were treated properly. It is possible that RIS monotherapy also contributed to this improvement.

It has been reported that cases with selective attention dysfunction have nonintegrated symptoms like serious thinking disorder [12]. As an indicator of vulnerability to schizophrenia, it has been pointed out that schizophrenics have disorders of various aspects in attention including selective attention [23]. Individuals with schizophrenia are known to have lower grades than healthy subjects in tasks that require working memory, such as the delayed response task, the target replacement task, and the $n$-back memory span task, which is the most complex task of all the memory span tasks [24]. It is thought that working memory is related to the functioning of the dorsolateral prefrontal cortex in schizophrenia. We have known that the individuals with schizophrenia have inadequate enforcement activity at issue in this area. Green et al. randomly assigned 59 cases with treatment-resistant schizophrenia into 2 groups (RIS group and haloperidol group) and performed DSDT. The authors showed that only the RIS group score improved significantly and that this effect had no relation with the anti-Parkinson drug and improvement of psychiatric symptoms [25]. Taking this report into consideration, we performed maintenance treatment using only RIS in our study. Thus, it is possible that the cases' DSDT scores improved as time passed. It is known that improvement of verbal working memory gives cases the advantage of acquiring skills for the future [26]. Therefore, in the SST group, the effect of SST appears to aid in concentration training and heightens self-efficacy, and the DSDT score improved significantly as a synergistic effect with medication in the SST group.

In the cases described above, SST enabled individuals with schizophrenia to acquire these skills and enhanced their social life. In parallel with these social improvements, DSDT improvements were obtained. When selective attention or working memory is disturbed, problems arise in actions of daily life, for example, when speaking with someone in a noisy environment and working recalling requests that someone asked just before. This study showed that SST might not only enable individuals with schizophrenia to acquire the skills needed directly for their social life, but also to enhance their selective attention and working memory through learned behaviors $[27,28]$.

\subsection{Improvement of Cognitive Function by SST}

Because cognitive function is not a direct target of SST, there is no research on interventions that treat cognitive function as direct outcome indices. A metanalysis by Kurtz et al. do not deal with cognitive function and neither do other metanalyses dealing with SST [19]. We searched the terms "cognitive functioning" and "social skills training" or "cognitive behavior therapy" in Medline, but no papers were found. In the Ichushi Service, the result was the same. As far as we know, this research has also not been mentioned in other reports or abstracts.

Ikebuchi et al. performed a total of 20 interventions that combined SST and psycho education in order to improve cognition in schizophrenia, and compared 3 groups (intervention-atypical psychotropics group, interventionconventional psychotropics group, and non-interventionatypical psychotropics group) using a quasi-experimental design. The cognitive function test, the digit span of WAIS-R, Trail Making test A and B, and word fluency and concepts fluency test were used. Attention function, which was evaluated by the digit span, showed significant improvement in the 2 groups using atypical psychotropics, but cognitive function was not improved by the intervention [29].

Granholm et al. [30] randomly separated 76 middleaged individuals with schizophrenia into 2 groups and performed 2-hour intervention 24 times combining cognitive behavior therapy and SST in the experimental group. This intervention improved cognitive function and intervened in cognitive content. This intervention aimed to enhance learning ability by using 3 modules: 1) Modules aimed at cognitive modification, studying the relationship between cognition and emotion and behavior, targeting positive symptoms. For middle age cases they offered the measures compensating for memory; 2) modules of training for communication skills for asking for help; 3 ) modules of program-solving training, which had 4 sessions for 2 periods. The result was that social activity and insight of disease increased in the experimental group, but there was no significant difference between the experimental group and the control group (conventional outpatient treatment). They evaluated the cognitive function by using various neuropsychological tests and they found that there was no significant difference in the general evaluation, executive, attention and vigilance, verbal learning and memory, and processing speed. In this intervention, target social functions and insight of disease were improved by SST in consideration of cognitive function, while cognitive function itself was not improved, and social function was not improved due to the 
improvement of cognitive function. Because individuals with mental disorder who were 42 - 74 years old were selected in this study, it is possible that it was difficult to improve the cognitive function. Of all rating scales of social function and skills, only a few scales were improved. Thus, there might be a limitation of this study due to aging or that the intervention was insufficient [30].

As mentioned above, there has been no report in the improvement of cognitive function by direct SST effect. Therefore, although the number of cases was small in our study, we showed the possibility of improved cognitive function by using learning experiences that need attention, working memory, and improvement of social life.

\subsection{Improvement of Other Rating Scales}

The SDSS score improved first, and a few items of LASMI improved later. By SST, improvement of subjective feeling of health and part of cognitive function led to, or maintained, an improvement of social function. The reasons why the subjective rating scales, such as SF36 and JSQLS, did not improve are as follows: 1) In the SST group, as 2 cases described above, although social life improved as time passed, subjective evaluation did not improve because of the increase in social stimuli and stress; 2) Because awareness of reality was improved according to the improvement of the cognitive function and neurologic manifestation, subjective evaluation became low. In fact, some previous studies have shown that there is no correlation between subjective QOL evaluation and objective QOL evaluation in schizophrenia [31].

\section{LIMITATIONS}

The limitation of this study is that the number of cases was small. As a result, there were only a few items that differed significantly. This is because the statistical power of the test may have been limited. There remains room for further investigation to clarify the results of this study.

\section{ACKNOWLEDGEMENTS}

The authors wish to thank all the staff in the PPST group and individuals with schizophrenia who cooperatively participated in this study.

\section{REFERENCES}

[1] Nishizono, M. (2012) The role of Pyschopharmacotherapy in the whole treatment structure. Seisinka Rinsyo Service, 12, 6-10.

[2] Liberman, R.P. (2008) Recovery from disability: Manual of psychiatric rehabilitation. American Psychiatric Publishing, Washington, DC.

[3] Lehman, A.F. (2003) The Schizophrenia Patient Out- comes Research Team (PORT): Updated treatment recommendations. Schizophrenia Bulletin, 30, 193-217. doi:10.1093/oxfordjournals.schbul.a007071

[4] Miyata, M., Fujii, Y., Inagaki, A., Inada, T. and Yagi, G. (1995) Reliability of the Japanese version of Brief Psychiatric Rating Scale (BPRS). Clinical Evaluation, 23, 357-367.

[5] Overall, J.E. and Gorham, D.R. (1988) The brief psychiatric rating scale (BPRS): Recent developments in ascertainment and scaling. Psychopharmacology Bulletin, 24, 97-99.

[6] Inada, T. and Yagi, G. (1996) Current topics in neuroleptic-induced extrapyramidal symptoms in Japan. The Keio Journal of Medicine, 45, 95-99. doi:10.2302/kjm.45.95

[7] Inada, T., Yagi, G. and Miura, S. (2002) Extrapyramidal symptom profiles in Japanese patients with schizophrenia treated with olanzapine or haloperidol. Schizophrenia Research, 57, 227-238. doi:10.1016/S0920-9964(01)00314-0

[8] Inada, T., Beasley, C., Tanaka, Y. and Walker, D.J. (2003) Extra-pyramidal symptom profiles assessed with the Drug-Induced Extrapyramidal Symptom Scale: Comparison with western scales in the clinical double-blind studies of schizophrenic patients treated with either olanzapine or haloperidol. International Clinical Psychopharmacology, 18, 39-48. doi:10.1097/00004850-200301000-00007

[9] Iwasaki, S., et al. (1994) The invention of life assessment scale for the mentally ill and its significance. Archives of Psychiatric Diagnostic Clinical Evaluation, 5, 221-231.

[10] Iwasaki, S., Miyauchi, M., Oshima, I., Honda, M. and Hata, T. (1994) The development of Life Assessment Scale for the Mentally Ill: An assessment of the reliability. Clinical Psychiatry, 36, 1139-1151.

[11] Bowen, L., Wallace, C.J., Glynn, S.M., Nuechterlein, K.H., Lutzker, J.R. and Kuehnel, T.G. (1994) Schizophrenic individuals' cognitive functioning and performance in interpersonal interactions and skills training procedures. Journal of Psychiatric Research, 28, 289-301. doi:10.1016/0022-3956(94)90012-4

[12] Perry, W. and Braff, D.L. (1994) Information-processing deficits and thought disorder in schizophrenia. The American Journal of Psychiatry, 151, 363-367. doi:10.1016/0920-9964(95)95565-Q

[13] Fukuhara, S., Bito, S., Green, J., Hsiao, A. and Kurokawa, K. (1998) Translation, adaptation, and validation of the SF-36 Health Survey for use in Japan. Journal of Clinical Epidemiology, 51, 1037-1044. doi:10.1016/S0895-4356(98)00095-X

[14] Fukuhara, S., Ware, J.E., Kosinski, M., Wada, S. and Gandek, B. (1998) Psychometric and clinical tests of validity of the Japanese SF-36 Health Survey. Journal of Clinical Epidemiology, 51, 1045-1053. doi:10.1016/S0895-4356(98)00096-1

[15] Ware, J.E. and Sherbourne, C.D. (1992) The MOS 36-item short form health survey (SF-36) I. Conceptual framework and item selection. Medical Care, 30, 473483. doi:10.1097/00005650-199206000-00002

[16] Bitter, I., Jaeger, J., Agdeppa, J. and Volavka, J. (1989) 
Subjective symptoms: Part of the negative syndrome of schizophrenia? Psychopharmacology Bulletin, 25, 180-184.

[17] Jaeger, J., Bitter, I., Czobor, P. and Volavka, J. (1990) The measurement of subjective experience in schizophrenia: The Subjective Deficit Syndrome Scale. Comprehensive Psychiatry, 31, 216-226. doi:10.1016/0010-440X(90)90005-D

[18] Kokai, M., Inada, T., Ohara, K., Shimizu, M., Iwao, H. and Morita, Y. (2003) Inter-rater and test retest reliability of the Japanese version of the subjective deficit syndrome scale. Human Psychopharmacology: Clinical and Experimental, 18, 145-149. doi:10.1002/hup.439

[19] Kurtz, M.M. and Mueser, K.T. (2008) A meta-analysis of controlled research on social skills training for schizophrenia. Journal of Consulting and Clinical Psychology, 76, 491-504. doi:10.1016/j.schres.2007.12.124

[20] Emmerson, L.C., Granholm, E., Link, P.C., McQuaid, J.R. and Jeste, D.V. (2009) Insight and treatment outcome with cognitive-behavioral social skills training for older people with schizophrenia. Journal of Rehabilitation Research and Development, 46, 1053-1058. doi:10.1682/JRRD.2009.04.0042

[21] Kopelowicz, A., Liberman, R.P. and Zarate, R. (2006) Recent advances in social skills training for schizophrenia. Schizophrenia Bulletin, 32, S12-S23. doi: $10.1093 / \mathrm{schbul} / \mathrm{sbl} 023$

[22] Oltmanns, T.F. and Neale, J.M. (1975) Schizophrenic performance when distracters are present: Attentional deficit or differential task difficulty? Journal of Abnormal Psychology, 84, 205-209. doi:10.1037/h0076721

[23] Cornblatt, B., Obuchowski, M., Roberts, S., Pollack, S. and Erlenmeyer-Kimling, L. (1999) Cognitive and behavioral precursors of schizophrenia. Development and Psychopathology, 11, 487-508. doi:10.1017/S0954579499002175
[24] Gold, J.M., Carpenter, C., Randolph, C., Goldberg, T.E. and Weinberger, D.R. (1997) Auditory working memory and Wisconsin Card Sorting Test performance in schizophrenia. Archives of General Psychiatry, 54, 159-165. doi:10.1001/archpsyc.1997.01830140071013

[25] Green, M.F., et al. (1997) Does risperidone improve verbal working memory in treatment-resistant schizophrenia? American Journal of Psychiatry, 154, 799-804.

[26] Green, M.F. (1996) What are the functional consequences of neurocognitive deficits in schizophrenia? American Journal of Psychiatry, 153, 321-330.

[27] Hatashita-Wong, M. and Silverstein, S.M. (2003) Coping with voices: Selective attention training for persistent auditory hallucinations in treatment refractory schizophrenia. Psychiatry, 66, 255-261. doi:10.1521/psyc.66.3.255.25162

[28] Tyson, P.J., Laws, K.R., Flowers, K.A., Tyson, A. and Mortimer, A.M. (2006) Cognitive function and social abilities in patients with schizophrenia: Relationship with atypical antipsychotics. Psychiatry and Clinical Neurosciences, 60, 473-479. doi:10.1111/j.1440-1819.2006.01534.x

[29] Ikebuchi, E., Suzuki, H., Andou, Y., Ando, Y., Numaguchi, R. and Kmura, M. (2007) The effect of atypical antipsychotropic to effectiveness of program to improve of deficit insight in schizophrenics. Seisinigaku, 49, 607-617.

[30] Granholm, E., et al. (2005) A randomized controlled trial of cognitive behavioral social skills training for middleaged and older outpatients with chronic schizophrenia. American Journal of Psychiatry, 162, 520-529. doi:10.1176/appi.ajp.162.3.520

[31] Osada, H., et al. (1998) Quality of life in schizophrenic patients (II). Tokyo Academy of Health Sciences, 1, 107110. 